Meta

Journal des traducteurs

Translators' Journal

\title{
Liste des relecteurs 2019
}

Volume 64, numéro 3, décembre 2019

URI : https://id.erudit.org/iderudit/1070528ar

DOI : https://doi.org/10.7202/1070528ar

Aller au sommaire du numéro

Éditeur(s)

Les Presses de l’Université de Montréal

ISSN

0026-0452 (imprimé)

1492-1421 (numérique)

Découvrir la revue

Citer ce document

(2019). Liste des relecteurs 2019. Meta, 64(3), 575-576.

https://doi.org/10.7202/1070528ar

Ce document est protégé par la loi sur le droit d'auteur. L'utilisation des services d'Érudit (y compris la reproduction) est assujettie à sa politique d'utilisation que vous pouvez consulter en ligne.

https://apropos.erudit.org/fr/usagers/politique-dutilisation/
Cet article est diffusé et préservé par Érudit.

Érudit est un consortium interuniversitaire sans but lucratif composé de l’Université de Montréal, l'Université Laval et l'Université du Québec à Montréal. Il a pour mission la promotion et la valorisation de la recherche. https://www.erudit.org/fr/ 


\section{Liste des relecteurs 2019}

Nous remercions chaleureusement toutes celles et ceux qui ont donné de leur temps et qui ont contribué à la revue. Toute omission est involontaire de notre part; veuillez nous la signaler.

Marco Agnetta (Universität Hildesheim)

Icíar Alonso Araguás (Universidad de Salamanca)

Gemma Andújar Moreno (Universitat Pompeu Fabra)

María Inés Arrizabalaga (Universidad Nacional de Córdoba)

Brian James Baer (Kent State University)

María Teresa Bajo Molina (Universidad de Granada)

Christian Balliu (Haute École de Bruxelles)

Salah Basalamah (Université d'Ottawa)

Jeremy L. Brunson (St. Catherine University)

Joost Buysschaert (Universiteit Gent)

María Calzada Pérez (Universitat Jaume I)

Ovidi Carbonell Cortés (Universidad de Salamanca)

Raymond Chakhachiro (University of Western Sydney)

Andrew Chesterman (University of Helsinki)

Marco Civico (Université de Genève)

Alice Colombo (University of Bristol)

Michael Cronin (Trinity College Dublin)

Carmen Cuéllar Lázaro (Universidad de Valladolid)

Daria Dayter (Universität Basel)

Xanthippi Dimitroulia (Aristotle University of Thessaloniki)

Álvaro Echeverri (Université de Montréal)

Jo-Anne Elder (chercheuse indépendante)

Alberto Fernández Costales (Universidad de Oviedo)

Óscar Ferreiro Vázquez (Universidade de Vigo)

Valérie Florentin (Université de Hearst)

Lynne Franjié (Université Charles-de-Gaulle Lille 3)

Montserrat Franquesa Gòdia (Universitat Autònoma de Barcelona)

Fang Gao (Nanjing University)

Nikolay Garbovskiy (Lomonosov Moscow State University)

Xoán Manuel Garrido Vilariño (Universidade de Vigo)

Anna Ghiglione (Université de Montréal)

Patricia Godbout (Université de Sherbrooke)

Gabriel González Núñez (University of Texas Rio Grande Valley)

Ting Guo (University of Exeter)

Kaibao Hu (Shanghai Jiao Tong University)

Moira Inghilleri (University of Massachusetts Amherst)

Magda Jeanrenaud (Universitatea „Alexandru Ioan Cuza” din Iași)

Meta LXIV, 3, 2019 
Meng Ji (The University of Sydney)

Esmaeil Kalantari (Université de Montréal)

Dorothy Kenny (Dublin City University)

Marja Kivilehto (Tampereen yliopisto)

Aurelia Klimkiewicz (York University)

Jane Koustas (Brock University)

Alexandra Krause (Universität Wien)

Michèle Laliberté (Université du Québec en Outaouais)

Gillian Lane-Mercier (McGill University)

Antonio Lavieri (Università Degli Studi Di Palermo)

Judith Lavoie (Université de Montréal)

René Lemieux (Université de Sherbrooke)

Defeng Li (University of Macau)

Rosa Elvira Luna García (Universidad Femenina del Sagrado Corazón)

Sarah Maitland (Goldsmiths, University of London)

Oumarou Mal Mazou (Université de Liège)

Anne Malena (University of Alberta)

Marina Manfredi (Università di Bologna)

Maialen Marin-Lacarta (Hong Kong Baptist University)

Marta Mateo Martínez-Bartolomé (Universidad de Oviedo)

Jean-Guy Mboudjeke (University of Windsor)

Julie McDonough Dolmaya (York University)

Elena Meteva-Rousseva (Sofia University)

John Milton (Universidade de São Paulo)

Christian Olalla Soler (Universitat Autònoma de Barcelona)

Mehtap Özdemir (University of Massachusetts Amherst)

Marina Ramos Caro (Universidad de Murcia)

Alessandra Riccardi (Università degli Studi di Trieste)

Wes Robertson (Macquarie University)

Cynthia Roy (Gallaudet University)

María Ángeles Ruiz Moneva (Universidad de Zaragoza)

Mohammad Saleh Sanatifar (Tabaran Institute of Higher Education)

José Santaemilia Ruiz (Universitat de València)

Teresa Seruya (Universidade de Lisboa)

Erich Steiner (Universität des Saarlandes)

Esli Struys (Vrije Universiteit Brussel)

Yi-feng Sun (University of Macau)

Agnieszka Szarkowska (University of Warsaw)

Kayvan Tahmasebian (chercheur indépendant)

Marija Todorova (Hong Kong Polytechnic University)

Marie-Hélène Catherine Torres (Universidade Federal de Santa Catarina)

Ira Torresi (Università di Bologna)

Margarida Vale de Gato (Universidade de Lisboa)

Luc van Doorslaer (Katholieke Universiteit Leuven)

Sylvie Vandaele (Université de Montréal)

George Varsos (National and Kapodistrian University of Athens)

Judith Woodsworth (Concordia University)

Jun Xu (Nanjing University)

Yan Ying (University of Leicester)

Danielle Zaslavsky Rabinovici (El Colegio de México) 